\title{
A Gauss-Bonnet formula for moduli spaces of Riemann surfaces
}

\author{
Enrico Leuzinger
}

\begin{abstract}
We prove a Gauss-Bonnet theorem for (finite coverings of) moduli spaces of Riemann surfaces endowed with the McMullen metric. The proof uses properties of an exhaustion of moduli spaces by compact submanifolds with corners and the Gauss-Bonnet formula of Allendoerfer and Weil for Riemannian polyhedra.
\end{abstract}

Key words: Moduli spaces of Riemann surfaces, Mapping class groups, Euler-Poincaré characteristic, Gauss-Bonnet theorems.

2000 MSC: 32G15, 53C20;

\section{Introduction}

Mapping class groups (acting on Teichmüller spaces) and arithmetic subgroups of semisimple Lie groups (acting on symmetric spaces) are prominent objects investigated in geometric group theory. In the last four decades several authors - beginning with W. Harvey [16], J. Harer [15] and N. Ivanov [18] - have discovered remarkable similarities (but also differences) between these two classes of groups (see e.g. [20], Section 9 for a discussion and also the more recent [2], [11] and [21]).

The chief goal of the present paper is to add a further item to the list of similarities: the Gauss-Bonnet formula. We consider a compact, orientable surface of genus $g$ with $p$ punctures $S=S_{g, p}$ such that $3 g-3+p>0$. The mapping class $\operatorname{group} \operatorname{Mod}(S)$ of $S$ acts properly discontinuously on Teichmüller space $\mathscr{T}(S)$. Let $\Gamma$ be a torsion-free, finite index subgroup of $\operatorname{Mod}(S)$. Then the Euler-Poincaré characteristic of $\operatorname{Mod}(S)$ is defined as

$$
\chi(\operatorname{Mod}(S)):=[\operatorname{Mod}(S): \Gamma]^{-1} \chi(\Gamma \backslash \mathscr{T}(S))
$$


Equivalently $\chi(\operatorname{Mod}(S))$ can be interpreted as the orbifold Euler characteristic of the moduli space of Riemann surfaces $\operatorname{Mod}(S) \backslash \mathscr{T}(S)$. J. Harer and D. Zagier [14] showed that for $g>1$

$$
\chi\left(\operatorname{Mod}\left(S_{g, 1}\right)\right)=\zeta(1-2 g) \text { and also } \chi\left(\operatorname{Mod}\left(S_{g, 0}\right)\right)=\frac{1}{2-2 g} \zeta(1-2 g)
$$

where $\zeta(s)$ is the Riemann zeta function. Note that $\zeta(1-2 g)=-B_{2 g} / 2 g \in \mathbb{Q}$ where $B_{2 g}$ is the $2 g$-th Bernoulli number.

This result should be compared with the Euler-Poincaré characteristic of arithmetic groups. For the integer symplectic group, for instance, a general result of G. Harder implies

$$
\chi(S p(2 n, \mathbb{Z}))=\prod_{k=1}^{n} \zeta(1-2 k)=\prod_{k=1}^{n}\left(-B_{2 k} / 2 k\right),
$$

see [13] or [30], Section 3. Harder proved a Gauss-Bonnet theorem by which the computation of the Euler characteristic of an arithmetic subgroup $\Lambda$ of a semisimple Lie group $G$ becomes equivalent to the computation of the volume of the locally symmetric space $\Lambda \backslash G / K$. The proof strongly relies on the reduction theory of arithmetic groups due to A. Borel and Harish-Chandra. In [25] I developped an analogous theory for mapping class groups based on work of Ivanov. This raises the question whether a Gauss-Bonnet theorem also holds in the setting of mapping class groups. In contrast to the symmetric case, where there is an essentially unique left-invariant metric, there is no canonical metric on Teichmüller resp. moduli space. However, as we will see, the Kähler hyperbolic complete metric constructed by C. McMullen in [27] has sufficiently nice properties such that a Gauss-Bonnet formula holds.

The generalized Gauss-Bonnet theorem of C.B. Allendoerfer, A. Weil and S.S. Chern asserts that the Euler characteristic of a closed Riemannian manifold $(M, g)$ of even dimension $n$ is given by

$$
\chi(M)=\int_{M} \omega_{g}=\int_{M} \Psi_{g} \mathrm{dvol}_{g}
$$

where the Gauss-Bonnet-Chern form $\omega_{g}=\Psi_{g}$ dvol $_{g}$ is (locally) computable from the metric $g$. More precisely, let $\left\{\left.\frac{\partial}{\partial y_{k}}\right|_{p} ; 1 \leq k \leq n\right\}$ be an orthonormal frame field in a neighbourhood of $p \in M$ and let

$$
R_{i j k l}(p):=g\left(\left.R\left(\left.\frac{\partial}{\partial y_{k}}\right|_{p},\left.\frac{\partial}{\partial y_{l}}\right|_{p}\right) \frac{\partial}{\partial y_{j}}\right|_{p},\left.\frac{\partial}{\partial y_{i}}\right|_{p}\right)
$$

be the components of the curvature tensor, then

$$
\Psi_{g}(p)=\frac{1}{(2 \pi)^{n / 2} 2^{n}(n / 2) !} \sum_{\mu, v} \operatorname{sign}(\mu) \operatorname{sign}(v) R_{\mu_{1} \mu_{2} v_{1} v_{2}}(p) R_{\mu_{3} \mu_{4} v_{3} v_{4}}(p) \ldots R_{\mu_{n-1} \mu_{n} v_{n-1} v_{n}}(p)
$$


where $\mu$ and $v$ independently run through all permutations of $\{1,2, \ldots, n\}$, see [3], [6] or [31], Chapter 13 for more details.

Note that moduli spaces are even dimensional but not compact. However, with respect to the McMullen metric they have bounded sectional curvature and finite volume (see [27]). The Gauss-Bonnet theorem for open complete Riemannian manifolds with bounded sectional curvature and finite volume has been investigated by J. Cheeger and M. Gromov. They in particular showed that such manifolds $M$ admit exhaustions by compact manifolds with smooth boundary, $M_{i}$, such that $\operatorname{Vol}\left(\partial M_{i}\right) \rightarrow 0 \quad(i \rightarrow \infty)$ and for which the second fundamental forms II $\left(\partial M_{i}\right)$ are uniformly bounded (see [7], [8], [9] and also [12] 4.5.C). By a formula of Chern one has $\chi\left(M_{i}\right)=\int_{M_{i}} \omega_{g}+\int_{\partial M_{i}} \eta_{i}$ where $\eta_{i}$ is a certain form on the boundary $\partial M_{i}$ (see [6]). The above two properties imply that $\lim _{i \rightarrow \infty} \int_{\partial M_{i}} \eta_{i}=0$ and hence that $\chi\left(M_{i}\right)=\int_{M} \omega_{g}$ for sufficiently large $i$. As a consequence the Gauss-Bonnet theorem holds provided $\chi\left(M_{i}\right)=\chi(M)$ for all sufficiently large $i$. This last property is not necessarily true for the exhaustions guaranteed by [7]. It follows however, if in addition $\pi_{1}(M)$ is residually finite, the injectivity radius of the universal covering space has a positive lower bound and $M$ is of finite topological type (see [7], Thm. 3.1).

Locally symmetric spaces of higher rank yield important examples for the above class considered by Cheeger-Gromov. For such (arithmetic) quotients the Gauss-Bonnet formula was first proved by Harder (see [13]). In [22] I gave a new, simpler proof based on an exhaustion $V=\bigcup_{s \geq 0} V(s)$ of locally symmetric spaces by polyhedra, i.e. compact submanifolds with corners, and such that each polyhedron $V(s)$ is a strong deformation retract of $V$ (see [23]). The essential new feature of this exhaustion, which simplified the proof, is that the boundaries of $\partial V(s)$ consist of subpolyhedra of $V(s)$ which are projections of pieces of horospheres in the universal covering space of $V$. As a consequence their second fundamental forms are uniformly bounded and a formula of Allendoerfer-Weil from the 1940s applies.

Coming back to moduli spaces of Riemann surfaces, we note that the (orbifold-) fundamental group of moduli space is the mapping class group, which is known to be residually finite (see [19]). Moreover, with respect to the McMullen metric, the injectivity radius of the universal covering space (i.e., Teichmüller space) has a positive lower bound (see [27]) and finally moduli space is of finite topological type (see e.g. Lemma 1 below). Thus by the result of Cheeger-Gromov refered to above, a Gauss-Bonnet formula holds. The goal of the present note is to give a new, independent proof of this fact. In contrast to the general situations considered by Cheeger and Gromov there is additional structural information available (as in the in the case of locally symmetric spaces). This allows us to avoid the rather involved technical arguments and constructions in [8]. 
Theorem (Gauss-Bonnet for moduli spaces) Let $S=S_{g, p}$ be a compact, orientable surface of genus $g$ with $p$ punctures such that $3 g-3+p>0$. Let $\Gamma \subset \operatorname{Mod}(S)$ be a torsion free, finite index subgroup of the mapping class group of $S$ consisting of pure elements and let $\mathscr{M}(S)=\Gamma \backslash \mathscr{T}(S)$ be the corresponding finite covering space of the moduli space of Riemann surfaces $\operatorname{Mod}(S) \backslash \mathscr{T}(S)$. Let $\omega_{g_{M}}=\Psi_{g_{M}} \mathrm{dvol}_{g_{M}}$ be the Gauss-Bonnet-Chern form associated to the McMullen metric $g_{M}$ on $\mathscr{M}(S)$ and let $\chi(\mathscr{M}(S))$ be the Euler-Poincaré characteristic of $\mathscr{M}(S)$. Then the following Gauss-Bonnet formula holds:

$$
\chi(\mathscr{M}(S))=\int_{\mathscr{M}(S)} \omega_{g_{M}}=\int_{\mathscr{M}(S)} \Psi_{g_{M}} \mathrm{dvol}_{g_{M}} .
$$

The proof is modelled on the one for locally symmetric spaces in [22]. We use an exhaustion of moduli spaces by compact submanifolds with corners due to Ivanov [18] whose properties were further studied in [25]. As already mentioned above, moduli space has bounded sectional curvature and finite volume with respect to the McMullen metric. We will show in addition that the boundaries of the exhausting polyhedra have bounded second fundamental forms, so that we again can use [3].

Remarks. (1) There are several metrics on Teichmüller resp. moduli space which are quasi-isometric to the Teichmüller metric (see e.g. [26]). It is conceivable that a GaussBonnet formula also holds for certain of these other metrics (some of which seem to be more canonical from the point of view of possible applications).

(2) In principle, uniform bounds on the density $\Psi_{g_{M}}$ in the above Gauss-Bonnet formula can be used to estimate volumes of moduli spaces with respect to the McMullen (or Teichmüller) metric. Unfortunately, mere uniform curvature bounds $\left|R_{i j k l}(p)\right| \leq$ const. seem to be too coarse to provide interesting asymptotic volume information. Note that for the surfaces $S_{g, 0}$ the (Teichmüller) volume grows more than exponentially in $g$. In fact, Proposition 2 below and the remark following it together with Proposition 3 imply that it is at least $g$ !. This is similar to the volume growth of locally symmetric space with respect to dimension.

Notation. Explicit constants are irrelevant for our purpose. If $f$ and $g$ are positive real valued functions on a set $S$ we thus simply write $f \prec g$ if there is a constant $c>0$ such that $f(s) \leq c g(s)$ for all $s \in S$. Similarly, $f \asymp g$ if $f(s) \leq c_{1} g(s)$ and $g(s) \leq c_{2} f(s)$ for positive constants $c_{1}, c_{2}$.

Acknowledgement. I would like thank C.T. McMullen for helpful correspondence. 


\section{The formula of Allendoerfer and Weil}

A $C^{\infty}$-manifold with corners is a topological Hausdorff space locally modeled upon a product of lines and half-lines and such that coordinate changes are of class $C^{\infty}$. For precise definitions and basic information about this concept we refer to [10]. A Riemannian polyhedron is a compact manifold with corners equipped with a Riemannian metric.

Let $P^{n}$ be an $n$-dimensional Riemannian polyhedron with boundary consisting of a finite family of lower dimensional subpolyhedra $P_{E}^{n-k}(1 \leq k \leq n)$ and with Riemannian metric induced from $P^{n}$. The outer angle $O(p)$ at a point $p$ of $P_{E}^{n-k}$ is defined as the set of all unit tangent vectors $v \in T_{p} P^{n}$ such that $\langle v, w\rangle_{p} \leq 0$ for all $w$ in the tangent cone of $P^{n}$ at $p$. Note that $O(p)$ is a spherical cell bounded by "great spheres" in the $(k-1)$-dimensional unit sphere of the normal space of $P_{E}^{n-k} \subset P^{n}$ at $p$. In [3] Allendoerfer and Weil define a certain real valued function $\Psi_{E, k}$ on the outer angles of $P_{E}^{n-k}$. The explicit form of this function will not be needed in this paper. We shall only use the fact that $\Psi_{E, k}$ is locally computable from the components of the metric and the curvature tensor of $P^{n}$ and from the components of the second fundamental forms $\mathrm{II}_{Z}(p), Z \in O(p)$, of $P_{E}^{n-k}$ in $P^{n}$. Let $\Psi$ dvol denote the Gauss-Bonnet-Chern form on $P^{n}$ and $\mathrm{dvol}_{E}$ (resp. $d \omega_{k-1}$ ) the volume element of $P_{E}^{k}$ (resp. of the standard unit sphere $S^{k-1}$ ). The inner Euler characteristic $\chi^{\prime}$ of $P^{n}$ is by definition the Euler characteristic of the open complex consisting of all inner cells in an arbitrary simplicial subdivision of $P^{n}$.

The generalized Gauss-Bonnet formula of Allendoerfer-Weil for Riemannian polyhedra proved in [3] has then the following form:

Proposition 1 Let $P^{n}$ be a Riemannian polyhedron of even dimension $n$ with boundary consisting of a finite family of subpolyhedra $P_{E}^{n-k}$. Then the inner Euler characteristic of $P^{n}$ is given by

$$
\chi^{\prime}\left(P^{n}\right)=\int_{P^{n}} \Psi \mathrm{dvol}+\sum_{E} \sum_{k=1}^{n} \int_{P_{E}^{n-k}}\left(\int_{O(p)} \Psi_{E, k} d \omega_{k-1}\right) \operatorname{dvol}_{E}(p) .
$$

Remark. This formula has also been used recently by McMullen in [28] to compute the complex hyperbolic volume of moduli spaces of configurations of ordered points on the Riemann sphere. 


\section{A polyhedral exhaustion of moduli spaces}

\subsection{Moduli spaces and the complex of curves}

Let $S=S_{g, p}$ be a compact, orientable surface of genus $g$ with $p$ punctures such that $d(S):=$ $3 g-3+p>0$. This last assumption implies that $S$ carries finite volume Riemannian metrics of constant curvature -1 and $p$ cusps. A marked hyperbolic surface is a pair $(X,[f])$ where $X$ is a smooth surface equipped with a complete Riemannian metric of constant curvature -1 and where $[f]$ denotes the isotopy class of a diffeomorphism $f: X \longrightarrow S$ mapping cusps to punctures. Two marked surfaces $\left(X_{1},\left[f_{1}\right]\right)$ and $\left(X_{2},\left[f_{2}\right]\right)$ are equivalent if there is an isometry $h: X_{1} \longrightarrow X_{2}$ such that $\left[f_{2} \circ h\right]=\left[f_{1}\right]$. The collection of these equivalence classes is (one possible definition of) the Teichmüller space $\mathscr{T}(S)$ of $S$. The mapping class group $\operatorname{Mod}(S)$ is the group of all orientation preserving diffeomorphisms of $S$, which fix the punctures, modulo isotopies which also fix the punctures. The $\operatorname{group} \operatorname{Mod}(S)$ acts on $\mathscr{T}(S)$ according to the formula $h \cdot(X,[f]):=(X,[h \circ f])$, for $h \in \operatorname{Mod}(S),(X,[f]) \in \mathscr{T}(S)$. The corresponding orbit space $\operatorname{Mod}(S) \backslash \mathscr{T}(S)$ is the moduli space of isometry classes of hyperbolic surfaces (obtained by forgetting the marking).

The complex of curves $\mathscr{C}(S)$ of $S$ is an infinite (even locally infinite) simplicial complex of dimension $d(S)-1$. Note that $d(S)=\frac{1}{2} \operatorname{dim} \mathscr{T}(S)$. The vertices of $\mathscr{C}(S)$ are the isotopy classes of simple closed curves (called circles) on $S$, which are non-trivial (i.e., not contractible in $S$ to a point or to a component of $\partial S$ ). We denote the isotopy class of a circle $C$ by $\langle C\rangle$. A set of $k+1$ vertices $\left\{\alpha_{0}, \ldots \alpha_{k}\right\}$ spans a $k$-simplex of $\mathscr{C}(S)$ if and only if $\alpha_{0}=\left\langle C_{0}\right\rangle, \ldots, \alpha_{k}=\left\langle C_{k}\right\rangle$ for some set of pairwise non-intersecting circles $C_{0}, \ldots, C_{k}$. For a simplex $\sigma \in \mathscr{C}(S)$ we denote by $|\sigma|$ the number of its vertices. A crucial fact is that $\mathscr{C}(S)$ is a thick chamber complex. In particular every simplex is the face of a maximal simplex. Moreover the mapping class group $\operatorname{Mod}(S)$ acts simplicially on $\mathscr{C}(S)$ and the quotient $\operatorname{Mod}(S) \backslash \mathscr{C}(S)$ is a finite (orbi-) complex (see [16], Proposition 1).

We next consider finite index, torsion-free subgroups $\Gamma$ of $\operatorname{Mod}(S)$ which, in addition, consist of pure mapping classes. Recall that a mapping class $h \in \operatorname{Mod}(S)$ is called pure if it can be represented by a diffeomorphism $f: S \longrightarrow S$ fixing (pointwise) some union $\Lambda$ of disjoint and pairwise non-isotopic nontrivial circles on $S$ and such that $f$ does not permute the components of $S \backslash \Lambda$ and induces on each component of the cut surface $S_{\Lambda}$ a diffeomorphism isotopic to a pseudo-Anosov or to the identity diffeomorphism (see [20], §7.1). It is wellknown that such subgroups $\Gamma$ exist. For example one can take $\Gamma=\Gamma_{S}(m)$, the kernel of the natural homomorphism $\operatorname{Mod}(S) \longrightarrow \operatorname{Aut}\left(H_{1}(S, \mathbb{Z} / m \mathbb{Z})\right), m \geq 3$, defined by the action of diffeomorphisms on homology (see e.g. [20], §7.1). 


\subsection{A partition and exhaustion of moduli space}

Let $\Gamma \subset \operatorname{Mod}(S)$ be as in the previous section. Using work of N. Ivanov I constructed in [25] a $\Gamma$-invariant partition of Teichmüller space into disjoint subsets, which in turn yields a partition of $\mathscr{M}(S)=\Gamma \backslash \mathscr{T}(S)$. In order to formulate that result more precisely, we need length functions. Let $\alpha$ be a vertex of $\mathscr{C}(S)$, i.e., $\alpha=\langle C\rangle$ for a (non-trivial) circle $C$. Since $d(S)>0$, any point $X \in \mathscr{T}(S)$ represents a finite volume Riemann surface of curvature -1 with $p$ cusps. On the surface $X$ the isotopy class of $\alpha$ contains a unique geodesic loop; let $l_{\alpha}(X)$ denote its length. This defines a (smooth) function $l_{\alpha}: \mathscr{T}(S) \longrightarrow \mathbb{R}_{>0}$ for every vertex $\alpha \in \mathscr{C}(S)$. For $\varepsilon>0$ we then define the $\varepsilon$-thick part of Teichmüller space

$$
\operatorname{Thick}_{\varepsilon} \mathscr{T}(S):=\left\{X \in \mathscr{T}(S) \mid l_{\alpha}(X) \geq \varepsilon, \forall \alpha \in \mathscr{C}(S)\right\}
$$

This set is $\Gamma$-invariant and its quotient, Thick $\mathcal{E}_{\mathcal{M}}(S)$, is the $\mathcal{\varepsilon}$-thick part of $\mathscr{M}(S)=\Gamma \backslash \mathscr{T}(S)$.

For every vertex $\alpha \in \mathscr{C}(S)$ we further set $\mathscr{H}_{\varepsilon}(\alpha):=\left\{X \in \operatorname{Thick}_{\mathcal{\varepsilon}} \mathscr{T}(S) \mid l_{\alpha}(X)=\varepsilon\right\}$. Then (for fixed $\varepsilon$ sufficiently small) we have $\partial \operatorname{Thick}_{\varepsilon} \mathscr{T}(S)=\bigcup_{\alpha} \mathscr{H}_{\mathcal{\varepsilon}}(\alpha)$. Given $\sigma \in \mathscr{C}(S)$ we denote by $S_{\sigma}$ the corresponding cut surface, i.e., the result of cutting $S$ along (nonintersecting) circles from the isotopy classes $\alpha \in \sigma$. In [25] I proved

Proposition 2 Let $S=S_{g, p}$ be a compact, orientable surface of genus $g$ with p punctures such that $3 g-3+p>0$. Let $\Gamma$ be a torsion free, finite index subgroup of the mapping class group of $S$ consisting of pure elements and let $\mathscr{M}(S)=\Gamma \backslash \mathscr{T}(S)$ be the corresponding finite covering space of the moduli space of Riemann surfaces. Finally let $\mathscr{E}$ be the set of simplices of the finite simplicial complex $\Gamma \backslash \mathscr{C}(S)$.

Then following holds:

(1) There exists $\varepsilon=\varepsilon(S)>0$ such that there is a partition of $\mathscr{M}(S)$, i.e., a disjoint union

$$
\mathscr{M}(S)=\operatorname{Thick}_{\varepsilon} \mathscr{M}(S) \sqcup \bigsqcup_{\sigma \in \mathscr{E}} \operatorname{Thin}_{\mathcal{\varepsilon}}(\mathscr{M}(S), \sigma)
$$

where $\operatorname{Thick}_{\mathcal{E}} \mathscr{M}(S)$ is a compact submanifold with corners and each $\operatorname{Thin}_{\mathcal{E}}(\mathscr{M}(S), \sigma)$ is diffeomorphic to $\mathscr{B}_{\varepsilon}(\sigma) \times \mathbb{R}_{>0}^{|\sigma|}$ with $\mathscr{B}_{\varepsilon}(\sigma)$ a (trivial) torus bundle over the $\varepsilon$-thick part of moduli space of the cut surface $S_{\sigma}$ such that the length of each boundary circle is $\varepsilon$ :

$$
0 \rightarrow T_{\varepsilon}^{|\sigma|} \rightarrow \mathscr{B}_{\varepsilon}(\sigma) \rightarrow \text { Thick }_{\varepsilon} \mathscr{M}\left(S_{\sigma}\right) \rightarrow 0
$$

(2) Each boundary face $\mathscr{B}_{\varepsilon}(\sigma)$, for $\sigma \in \mathscr{E}$, of Thick $_{\varepsilon} \mathscr{M}(S)$ is the image of $\bigcap_{\alpha \in \sigma} \mathscr{H}_{\varepsilon}(\alpha)$ under the natural projection $\pi: \mathscr{T}(S) \longrightarrow \mathscr{M}(S)$. 
Remark. In a similar exhaustion for locally symmetric spaces, the thin parts are indexed by the simplices of the rational Tits building modulo the arithmetic group (see [24], Theorem $\mathrm{C})$. The number of cells in this (finite) quotient is rather small; for instance, for the locally symmetric space of principally polarized abelian varieties, $S p(2 n, \mathbb{Z}) \backslash S p(2 n, \mathbb{R}) / U(n)$, it is always one. In contrast, the corresponding quotient for moduli spaces is large: The number of maximal simplices in $\operatorname{Mod}(S) \backslash \mathscr{C}(S)$ equals the number of different pant decompositions of $S$, which is roughly $g$ ! for large $g$ (see [5], Theorem 3.5.3).

Proposition 2 yields the

Corollary 1 There exists $\varepsilon_{0}>0$ depending only on $S$, such that there is a $\Gamma$-invariant exhaustion of Teichmüller space $\mathscr{T}(S)=\bigcup_{\varepsilon \leq \varepsilon_{0}} \operatorname{Thick}_{\varepsilon} \mathscr{T}(S)$, which induces an exhaustion of $\mathscr{M}(S)$ by polyhedra, i.e., by compact submanifolds with corners: $\mathscr{M}(S)=\bigcup_{\varepsilon \leq \varepsilon_{0}} \operatorname{Thick}_{\mathcal{\varepsilon}} \mathscr{M}(S)$.

In contrast to some of the exaustions constructed by Cheeger-Gromov (see Section 1), the topology of the exhausting polyhedra does not change. Namely, we have

Lemma 1 For every sufficiently small $\varepsilon$ the moduli space $\mathscr{M}(S)$ is homeomorphic to the interior of the polyhedron $\mathrm{Thick}_{\mathcal{\varepsilon}} \mathscr{M}(S)$ in $\mathscr{M}(S)$, and $\mathrm{Thick}_{\mathcal{\varepsilon}} \mathscr{M}(S)$ is a strong deformation retract of $\mathscr{M}(S)$.

Proof. Recall that the complex of curves $\mathscr{C}(S)$ is a thick chamber complex. Given a simplex $\sigma \in \mathscr{C}(S)$ we can thus choose a simplex $\tau \in \mathscr{C}(S)$ of maximal dimension $d(S)-1=$ $3 g-4+p$ containing $\sigma$. Then, by [1], there are adapted Fenchel-Nielsen coordinates on Teichmüller space $\mathscr{T}(S)$, i.e., there is a diffeomorphism

$$
\Phi_{\tau}: \mathscr{T}(S) \longrightarrow \mathscr{T}\left(S_{\sigma}\right) \times \mathbb{R}^{|\sigma|} \times \mathbb{R}_{>0}^{|\sigma|} ; X \longmapsto(s(X), \theta(X), l(X)),
$$

where $s=\left(\theta_{\beta}, l_{\beta}\right)_{\beta \in \tau \backslash \sigma}$ parametrizes the Teichmüller space $\mathscr{T}\left(S_{\sigma}\right)$ of the cut surface $S_{\sigma}$, $\theta=\left(\theta_{\alpha}\right)_{\alpha \in \sigma} \in \mathbb{R}^{|\sigma|}$ are twist parameters and $l=\left(l_{\alpha}\right)_{\alpha \in \sigma} \in \mathbb{R}_{>0}^{|\sigma|}$ are length functions (here and elsewhere $|\sigma|$ denotes the number of vertices of $\sigma$ ).

We define the outer cone at $X \in \partial$ Thick $_{\mathcal{\varepsilon}} \mathscr{T}(S)$ as the preimage

$$
C O(X):=\Phi_{\tau}^{-1}\left\{\left(s(X), \theta(X),\left(l_{\alpha}\right)_{\alpha \in \sigma}\right) \mid l_{\alpha}<\varepsilon \text { for all } \alpha \in \sigma\right\} .
$$

By [25], Lemma 5, outer cones are $\Gamma$-invariant. This allows one to define outer cones in moduli space : for $X \in \operatorname{Thick}_{\varepsilon} \mathscr{M}(S)$ we set $C O(X):=\pi(C O(\hat{X}))$ where $\hat{X}$ is any lift of $X$. We then have (see [25], 2.2):

$$
\mathscr{M}(S)=\operatorname{Thick}_{\mathcal{E}} \mathscr{M}(S) \sqcup \bigsqcup_{X \in \partial \operatorname{Thick}_{\mathcal{E}} \mathscr{M}(S)} C O(X)=\text { Thick }_{\mathcal{\varepsilon}} \mathscr{M}(S) \sqcup \bigsqcup_{\sigma \in \mathscr{E}} \bigsqcup_{X \in \mathscr{B}_{\mathcal{\varepsilon}}(\sigma)} C O(X) .
$$


Note that each outer cone is diffeomorphic to the open hyperoctant $\mathbb{R}_{>0}^{|\sigma|}$. The claimed retraction is then given by retracting each outer cone to the apex of its closure.

\section{Estimates for the boundary subpolyhedra}

We wish to apply Proposition 1 to the polyhedra Thick $_{\mathcal{E}} \mathscr{M}(S)$ in the above exhaustion and then take the limit for $\varepsilon \rightarrow 0$. To that end we need uniform estimates for the second fundamental forms and the volumes of the (lower dimensional) boundary polyhedra with respect to the McMullen metric.

By Section 3, Proposition 2, we know that for every (sufficiently small) $\varepsilon$ the boundary polyhedra are of the form $\mathscr{B}_{\varepsilon}(\sigma)$ for $\sigma \in \mathscr{E}$, the set of simplices in the finite simplicial complex $\Gamma \backslash \mathscr{C}(S)$. Moreover, if $n:=\operatorname{dim} \mathscr{M}(S)$, we have $\operatorname{dim} \mathscr{B}_{\mathcal{E}}(\sigma)=n-|\sigma|$.

\subsection{An approximation of the metric on thin parts}

We will prove the Gauss-Bonnet formula with respect to the metric on moduli space constructed by McMullen in [27]. He showed that with respect to this metric $\mathscr{M}(S)$ is Kähler hyperbolic in the sense of Gromov and thus in particular carries a complete finite volume Riemannian metric of bounded sectional curvature. The McMullen metric is a modification of the (incomplete) Weil-Petersson metric and quasi-isometric to the Teichmüller metric (see [27]).

We next describe explicit representatives of the bi-Lipschitz class of the Teichmüller resp. McMullen metric on $(\varepsilon, \sigma)$-thin parts of $\mathscr{T}(S)$ (and $\mathscr{M}(S)$ ). To that end we use an expansion of the Weil-Petersson (WP) metric due to S. Wolpert (see [32], [33]).

For each length function $l_{\alpha}$ we set $u_{\alpha}:=-\log l_{\alpha}^{1 / 2}$. Considering this logarithmic root length instead of $l_{\alpha}$ itself is suggested by work of Wolpert (see e.g. [32], [33]). Following Wolpert we also set $\lambda_{\alpha}:=\operatorname{grad} l_{\alpha}^{1 / 2}\left(\operatorname{resp} . v_{\alpha}:=-\operatorname{grad} u_{\alpha}\right)$ and define the Fenchel-Nielsengauge as the differential 1-form $\rho_{\alpha}:=2 \pi\left(l_{\alpha}^{3 / 2}\left\langle\lambda_{\alpha}, \lambda_{\alpha}\right\rangle\right)^{-1}\left\langle, J \lambda_{\alpha}\right\rangle=2 \pi\left(\left\langle v_{\alpha}, v_{\alpha}\right\rangle\right)^{-1}\left\langle, J v_{\alpha}\right\rangle$ (see [33], 4.15). This gauge is normalized such that $l_{\alpha}\left(T_{\alpha}\right)=1$ for $T_{\alpha}:=(2 \pi)^{-1} l_{\alpha}^{3 / 2} J \lambda_{\alpha}$ the WP-unit infinitesimal FN angle variation. We also set $\tilde{\rho}_{\alpha}:=l_{\alpha}^{-1 / 2} \rho_{\alpha}$ (this gauge is normalized with respect to the Teichmüller-unit infinitesimal FN angle variation). In [25] I showed

Proposition 3 There is $\varepsilon_{*}>0$ depending only on $S$, such that for $\sigma \in \mathscr{C}(S), \varepsilon \leq \varepsilon_{*}$ and the 
$(\varepsilon, \sigma)$-thin part

$$
\operatorname{Thin}_{\varepsilon}(\mathscr{T}(S), \sigma):=\bigsqcup_{X \in \bigcap_{\alpha \in \sigma} \mathscr{H}_{\varepsilon}(\alpha)} C O(X) \cong \operatorname{Thick}_{\mathcal{\varepsilon}} \mathscr{T}\left(S_{\sigma}\right) \times \mathbb{R}^{|\sigma|} \times \mathbb{R}_{>0}^{|\sigma|}
$$

the following Finsler metric expansion of the Teichmüller (or McMullen) metric with respect to adapted FN-coordinates holds

$$
\|\cdot\|_{\mathscr{T}(S)}^{2} \asymp\|\cdot\|_{\mathscr{T}\left(S_{\sigma}\right)}^{2}+\sum_{\alpha \in \sigma} e^{-6 u_{\alpha}} \tilde{\rho}_{\alpha}^{2}+d u_{\alpha}^{2} .
$$

The bi-Lipschitz constants involved in this estimate only depend on $S$ and $\varepsilon_{*}$.

This metric approximation decends to the $(\varepsilon, \sigma)$-thin parts of moduli space (see [25], Cor. 3):

$$
\operatorname{Thin}_{\mathcal{\varepsilon}}(\mathscr{M}(S), \sigma):=\bigsqcup_{X \in \mathscr{B}_{\varepsilon}(\sigma)} C O(X) \cong \operatorname{Thick}_{\mathcal{\varepsilon}} \mathscr{M}\left(S_{\sigma}\right) \times T_{\varepsilon}^{|\sigma|} \times \mathbb{R}_{>0}^{|\sigma|}
$$

\subsection{Second fundamental forms of boundary polyhedra}

Lemma 2 Let $\varepsilon_{0}$ be so small that both Proposition 2 and Proposition 3 hold. Take $\varepsilon \leq \varepsilon_{0}$ and $\sigma \in \mathscr{E}$. Then the second fundamental forms of the boundary polyhedron $\mathscr{B}_{\varepsilon}(\sigma)$ with respect to outer angles in $\mathrm{Thick}_{\varepsilon} \mathscr{M}(S)$ are uniformly bounded by a constant independent of $\sigma$ and $\varepsilon$.

Proof. By Proposition 2(2) the boundary polyhedra are projections of intersections of level sets of length functions

$$
\mathscr{B}_{\varepsilon}(\sigma)=\pi\left(\bigcap_{\alpha \in \sigma} \mathscr{H}_{\varepsilon}(\alpha)\right) \text { where } \quad \mathscr{H}_{\varepsilon}(\alpha):=\left\{X \in \operatorname{Thick}_{\varepsilon} \mathscr{T}(S) \mid l_{\alpha}(X)=\varepsilon\right\}
$$

The outer angles (see Section 2) of $\mathscr{B}_{\varepsilon}(\sigma)$ are thus positive linear combinations of gradients of the lenght functions $l_{\alpha}$ for $\alpha \in \sigma$. Set $N_{\alpha}:=\frac{\operatorname{grad}\left(l_{\alpha}\right)}{\left\|\operatorname{grad}\left(l_{\alpha}\right)\right\|}$. Hence, to prove the Lemma, we have to show that (with respect to the McMullen metric)

$$
\left\langle D_{X} Y, N_{\alpha}\right\rangle=O(1) \text { for all } \alpha \in \sigma \text { and all unit tangent vectors } X, Y \text { of } \mathscr{B}_{\varepsilon}(\sigma) \text {. }
$$

We first note that the derivatives up to order two of $l_{\alpha}, \alpha \in \sigma$, are $O\left(l_{\alpha}\right)$. In fact, in the proof of Theorem 8.2. in [27] McMullen used the Bers embedding and euclidean polydiscs to show that the derivatives of $\log \left(l_{\alpha}\right)$ are $O(1)$ and hence the first dervatives of $l_{\alpha}$ are $O\left(l_{\alpha}\right)$ and similarly for second derivatives. 
We next show that $\left\|\operatorname{grad}\left(l_{\alpha}\right)\right\| \asymp l_{\alpha}$. Denote by $\operatorname{grad}_{*}\left(l_{\alpha}\right)$ the gradient of $l_{\alpha}$ with respect to the approximating product metric in Proposition 3 . Then for the adopted FN-coordinates we have $l_{\alpha}=e^{-2 u_{\alpha}}$ hence, if we denote by $\langle\cdot, \cdot\rangle_{*}$ the approximate metric, we have that $\operatorname{grad}_{*}\left(l_{\alpha}\right)$ is parallel to $\partial_{u_{\alpha}}$ and $\left\|\partial_{u_{\alpha}}\right\|_{*}=1$. Hence

$$
\left\|\operatorname{grad}_{*}\left(l_{\alpha}\right)\right\|_{*}=\left\langle\operatorname{grad}_{*}\left(l_{\alpha}\right), \partial_{u_{\alpha}}\right\rangle_{*}=d l_{\alpha}\left(\partial_{u_{\alpha}}\right)=-2 l_{\alpha}
$$

Since (on the thin part $\operatorname{Thin}_{\mathcal{E}}(\mathscr{M}(S), \sigma)$ under consideration) the McMullen metric is comparable to the approximate metric we eventually find

$$
\left\|\operatorname{grad}\left(l_{\alpha}\right)\right\| \asymp\left\|\operatorname{grad}_{*}\left(l_{\alpha}\right)\right\|_{*} \asymp l_{\alpha}
$$

By this estimate for the gradient, $\left\langle D_{X} Y, N_{\alpha}\right\rangle$ is $O(1)$ if $\left\langle D_{X} Y, \operatorname{grad}\left(l_{\alpha}\right)\right\rangle$ is $O\left(l_{\alpha}\right)$ for unit tangent vectors $X, Y$ of $\mathscr{B}_{\varepsilon}(\sigma)$. Now such an $Y$ is tangent to the level surface of $l_{\alpha}$ and thus $\left\langle Y, \operatorname{grad}\left(l_{\alpha}\right)\right\rangle=0$. This yields (see [29], Lemma 3.49)

$$
\left\langle D_{X} Y, \operatorname{grad}\left(l_{\alpha}\right)\right\rangle=-\left\langle Y, D_{X} \operatorname{grad}\left(l_{\alpha}\right)\right\rangle=-\operatorname{Hess}_{l_{\alpha}}(X, Y)=-(X Y) l_{\alpha}+\left(D_{X} Y\right) l_{\alpha}=O\left(l_{\alpha}\right)
$$

were the last estimate follows from the already mentioned estimates for the derivatives of order one and two and the additional fact that the covariant derivative $D_{X} Y$ is $O(1)$ for unit vectors $X, Y$ since the derivatives of the metric are also bounded (see again[27], Theorem $8.2)$.

\subsection{Volumes of boundary polyhedra}

We next estimate the volumes of the boundary polyhedra $\mathscr{B}_{\varepsilon}(\sigma)$ which have dimension $n-|\sigma|$, where $n=\operatorname{dim} \mathscr{M}(S)$.

Lemma 3 The $(n-|\sigma|)$-dimensional volume of each boundary subpolyhedron $\mathscr{B}_{\varepsilon}(\sigma)$ of Thick $_{\mathcal{E}} \mathscr{M}(S)$ satisfies

$$
\operatorname{Vol}\left(\mathscr{B}_{\varepsilon}(\sigma)\right) \prec \varepsilon,
$$

where the constants involved in this estimate are independent of $\sigma$ and $\varepsilon$.

Proof. Since boundedness of volume is a bi-Lipschitz invariant, we can work with the approximation of the McMullen metric from Proposition 3, On the thin part $\operatorname{Thin}_{\mathcal{E}}(\mathscr{M}(S), \sigma)$ the latter is comparable to a product of the McMullen metric on the thick part of the lowerdimensional moduli space $\mathscr{M}\left(S_{\sigma}\right)$ with a product of 2-dimensional hyperbolic metrics (the 
number of factors of the latter beeing equal to $|\sigma|)$. Now, by the general properties of the McMullen metric, the volume of $\mathscr{M}\left(S_{\sigma}\right)$ is bounded, hence in particular $\operatorname{Vol}\left(\operatorname{Thick}_{\mathcal{\varepsilon}} \mathscr{M}\left(S_{\sigma}\right)\right) \leq$ $c_{1}(\sigma)$. Similarly, we get for the torus fibre (see Proposition 2 (1)) from the expansion in Proposition 3

$$
\left.\operatorname{Vol}\left(T_{\varepsilon}^{\sigma}\right)\right) \leq c_{2}(\sigma) e^{-6(-\log \sqrt{\varepsilon})|\sigma|}=c_{2}(\sigma) \varepsilon^{3|\sigma|}
$$

Thus

$$
\left.\operatorname{Vol}\left(\mathscr{B}_{\varepsilon}^{\sigma}\right)\right) \leq c_{1}(\sigma) c_{2}(\sigma) \varepsilon^{3|\sigma|}
$$

Since the set $\mathscr{E}$ of simplices indexing the boundary polyhedra is finite and $\varepsilon \ll 1$, the claim of the Lemma follows.

\section{The proof of the Gauss-Bonnet formula}

In this section we give the proof of the Gauss-Bonnet formula for moduli spaces of Riemann surfaces equipped with the McMullen metric as stated in the introduction.

By Corollary 1 there is an exhaustion $\mathscr{M}(S)=\bigcup_{\varepsilon \leq \varepsilon_{0}}$ Thick $_{\varepsilon} \mathscr{M}(S)$ of $\mathscr{M}(S)$ by Riemannian polyhedra. Each polyhedron $\mathscr{M}(\varepsilon):=\operatorname{Thick}_{\mathcal{E}} \mathscr{M}(S)$ in this exhaustion is equipped with the Riemannian metric induced by the one of $\mathscr{M}(S)$. We set $n:=\operatorname{dim} \mathscr{M}(S)$ and $k:=|\sigma|$ (i.e. $\operatorname{dim} \mathscr{B}_{\varepsilon}(\sigma)=n-k$ ). Since $n$ is even Proposition 1 applied to $\mathscr{M}(\varepsilon)$ yields

$$
\left|\chi^{\prime}(\mathscr{M}(\varepsilon))-\int_{\mathscr{M}(\varepsilon)} \Psi \mathrm{dvol}\right| \prec \sum_{\sigma \in \mathscr{E}} \sum_{k=1}^{n} \int_{\mathscr{B}_{\mathcal{\varepsilon}}(\sigma)} \int_{O(X)}\left\|\Psi_{\sigma, k}\right\| d \omega_{k-1} \operatorname{dvol}_{\sigma}(X) .
$$

As remarked in Section 1, the function $\Psi_{\sigma, k}$ is locally computable from the components of the metric and the curvature tensor of $\mathscr{M}(\varepsilon)$ and from the components of the second fundamental forms of the $\mathscr{B}_{\varepsilon}(\sigma)$ in $\mathscr{M}(\varepsilon)$. The fact that the McMullen metric has bounded curvature together with Lemma 2 thus implies that $\left\|\Psi_{\sigma, k}\right\| \prec 1$ for all $\sigma, k$. Using Lemma 3 we conclude that

$$
\left|\chi^{\prime}(\mathscr{M}(\varepsilon))-\int_{\mathscr{M}(\varepsilon)} \Psi \mathrm{d} \operatorname{vol}\right| \prec \sum_{k, \sigma} \operatorname{Vol}\left(\mathscr{B}_{\varepsilon}(\sigma)\right) \prec \varepsilon
$$

By Lemma 1 we have $\chi^{\prime}(\mathscr{M}(\varepsilon))=\chi(\mathscr{M}(S))$ and since $\chi(\mathscr{M}(S))$ is an integer we have $\chi(\mathscr{M}(S))=\int_{\mathscr{M}(\varepsilon)} \Psi$ dvol for all sufficiently small $\varepsilon$. Since the polyhedra $\mathscr{M}(\varepsilon)$ exhaust $\mathscr{M}(S)$, the claimed formula follows. 


\section{References}

[1] W. AвIKOFF, The Real Analytic Theory of Teichmüller Space, Lecture Notes in Mathematics 820, Springer-Verlag, 1976.

[2] Arithmetic groups vs. mapping class groups: similarities, analogies and differences, Abstracts from the workshop held at MFO June 5-11, 2011. Oberwolfach reports 8 (2011), 1637-1708.

[3] C.B. Allendoerfer, A. Weil, The Gauss-Bonnet theorem for Riemannian polyhedra, Trans. Amer. Math. Soc. 53 (1943), 101-129.

[4] A. Borel, J.-P. Serre, Corners and arithmetic groups, Comment. Math. Helv., 48 (1973), 436-491.

[5] P. BUSER, Geometry and spectra of compact Riemann surfaces, Progress in Math. 106, Birkhäuser, 1992.

[6] S.S. CHERn, A simple intrinsic proof of the Gauss-Bonnet formula for closed Riemannian manifolds, Ann. of Math., 45 (1944), 747-752.

[7] J. Cheeger, M. Gromov, On the Characteristic Numbers of Complete Manifolds of Bounded Curvature and Finite Volume, in: Differential Geometry and Complex Analysis, H.E. Rauch Memorial Volume (I. Chavel, H.M. Farkas, Eds.), Berlin 1985, 115154.

[8] J. Cheeger, M. Gromov, Bounds on the von Neumann dimension of $L_{2}$ cohomology and the Gauss-Bonnet theorem for open manifolds, J. Diff. Geom. 21 (1985), 1-39.

[9] J. Cheeger, M. Gromov, Chopping Riemannian manifolds, in: A symposium in honour of M. do Carmo (B. Lawson, K. Tenenblat, Eds.), Pitman Monographs and Surveys in Pure and Applied Mathematics 52, New York 1991, 85-94.

[10] Douady, L. HÉrault, Arrondissement des variétés à coins, appendix of [4], 484489.

[11] B. FARB, A. LubotZKy, Y. Minski, Rank-1 phenomena for mapping class groups, Duke Math. J. 106 (2001), 581-597.

[12] M. Gromov, Volume and bounded cohomology, Publ. Math. IHES 56 (1982), 5-100. 
[13] G. HARDER, A Gauss-Bonnet formula for discrete arithmetically defined groups, Ann. scient. Éc. Norm. Sup. 4 (1971), 409-455.

[14] J. HARER, D. ZAGIER, The Euler characteristic of the moduli space of curves, Invent. math. 85 (1986), 457-485.

[15] J.L. HARER, The cohomology of the moduli space of curves. Theory of moduli (Montecatini Terme, 1985), 138-221, Lecture Notes in Math. 1337, Springer, Berlin, 1988.

[16] W.J. HARVEY, Boundary structure of the modular group, In: Riemann surfaces and related topics: Proceedings of the 1978 Stony Brook Conference (State Univ. New York, Stony Brook, 1978), pp. 245-251, Ann. of Math. Stud. 97, Princeton Univ. Press, Princeton, 1981.

[17] W.J. HARVEY, Remarks on the curve complex: classification of surface homeomorphisms, In: Kleinian Groups and Hyperblic 3-Manifolds, Lond. Math. Soc. LNS 299, 165-179, Cambridge Univ. Press, 2003.

[18] N.V. Ivanov, Complexes of curves and the Teichmüller modular group, Russ. Math. Surveys 42 (1987), 55-107.

[19] N.V. IVANOV, Subgroups of Teichmüller Modular Groups groups, Translations of Mathematical Monographs 115, AMS 1992.

[20] N.V. IVAnov, Mapping class groups. Handbook of geometric topology, 523-633, North-Holland, Amsterdam, 2002.

[21] L. Ji, A tale of two groups: arithmetic groups and mapping class groups, Handbook of Teichmüller theory. Vol. III, 157-295, IRMA Lect. Math. Theor. Phys. 17, Eur. Math. Soc., Zürich, 2012.

[22] E. LEUZINGER, On the Gauss-Bonnet formula for locally symmetric spaces of noncompact type, L'Ens. Math. 42 (1996), 201-214.

[23] E. LEUZINGER, On polyhedral retracts and compactifications of locally symmetric spaces, Diff. Geom. Appl. 20 (2004), 293-318.

[24] E. LEUZINGER, Tits geometry, arithmetic groups, and the proof of a conjecture of Siegel, J. Lie Theory 14 (2004), 317-338. 
[25] E. LEUZINGER, Reduction theory for mapping class groups and applications to moduli spaces, J. reine angew. Math. 649 (2010), 11-31.

[26] K. LiU, X. Sun, S.-T. Yau, Canonical metrics on the moduli space of Riemann surfaces I \& II, J. Diff. Geom. 68 (2004), 571-637 \& J. Diff. Geom. 69 (2005), 161-216.

[27] C.T. MCMullen, The moduli space of Riemann surfaces is Kähler hyperbolic, Ann. of Math. 151 (2000), 327-357.

[28] C.T. McMullen, The Gauss-Bonnet theorem for cone manifolds and volumes of moduli spaces, preprint 2013.

[29] B. O’NeILl, Semi-Riemannian Geometry, Academic Press, New York 1983.

[30] J.-P. SERRE, Arithmetic groups, In: Homological group theory, Lond. Math. Soc. LNS 36, 105-136, Cambridge Univ. Press, 1979.

[31] M. SPIVAK, A comprehensive introduction to differential geometry, Vol. 5, Publish or perish, Berkeley, 1979.

[32] S.A. WOLPERT, Geometry of the Weil-Petersson completion of Teichmüller space. Surveys in differential geometry, Vol. VIII (Boston, MA, 2002), 357-393, Int. Press, Somerville, MA, 2003.

[33] S.A. WOLPERT, Behaviour of geodesic length functions on Teichmüller space, J. Diff. Geom. 79 (2008), 277-334.

INSTITUTE FOR AlgebRa UND GEOMETRY

KARLSRUhe Institute OF TECHNOLOGy (KIT), GERMANY

enrico.leuzinger@kit.edu 